\title{
Interactive comment on "A comprehensive dataset of vegetation states, fluxes of matter and energy, weather, agricultural management, and soil properties from intensively monitored crop sites in Western Germany" by Tim G. Reichenau et al.
}

Anonymous Referee \#4

Received and published: 28 April 2020

I appreciate the authors' efforts in compiling this dataset. As a modeler, I understand the importance of such a dataset for model parameterization and validation. Data collection and compiling are usually two of the most time-consuming processes. It's good to see this dataset been organized following a consistent protocol. However, the importance of the current dataset is not clear. Such data (e.g. flux, managements) can be found from different sources. For example, Ameriflux (https://ameriflux.lbl.gov/) provides multi-year, quality-controlled data collected from hundreds of eddy-flux sites worldwide, and the management information for the cropland sites can be obtained 
from the website and the related publications. The authors did provide a description of the uniqueness of the dataset, but it sounds the data has limited impacts on at local area.

Honestly, I am also not very satisfied with many missing data (NAs) in this dataset, especially in the management information category. The dataset barely provides a three-year continuous management records. I understand that this is labor-intensive work, but the current dataset is relatively short-term coverage. Are the authors planning to continue the measurements and regularly update the dataset?

Another concern is the lack of land use history information. For example, land use change plays a pivotal role in altering soil property. How long have the sites been converted to cropland? Generally, conversion from natural vegetation to cropland triggers rapid decomposition of soil organic matters, which may last for decades. Have the lagged impacts from land use change gone? This background information is essential, which should be provided before the data can be used in modeling.

Interactive comment on Earth Syst. Sci. Data Discuss., https://doi.org/10.5194/essd-2019-193, 2020. 